\title{
Searches for Chiral Effects and Prospects for Isobaric Collisions at STAR/RHIC
}

\author{
Liwen Wen* \\ (for the STAR Collaboration) \\ University of California, Los Angeles \\ E-mail: lwen@physics.ucla.edu
}

\begin{abstract}
Searches for the chiral effects (the Chiral Magnetic Effect, the Chiral Vortical Effect and the Chiral Magnetic Wave) in heavy-ion collisions have been under theoretical and experimental investigations in recent years. A three-point correlator $\gamma$, has been used to measure electric charge separation across the reaction plane. Significant background sources arising from charge/momentum conservation coupled with the elliptic flow have also been identified. Isobaric collisions are proposed to potentially disentangle the flow related background and magnetic field induced charge separation signals in heavy-ion collisions. In these proceedings, we show recent STAR results on the $\gamma$ correlator using charged hadrons and identified particles in $200 \mathrm{GeV} \mathrm{Au+Au} \mathrm{collisions.}$ Moreover, the CME signal significance vs. background level is projected for the upcoming isobaric collisions $(\mathrm{Zr}+\mathrm{Zr}$ and $\mathrm{Ru}+\mathrm{Ru})$ in 2018 at $\mathrm{RHIC}$.
\end{abstract}

Critical Point and Onset of Deconfinement

7-11 August, 2017

The Wang Center, Stony Brook University, Stony Brook, NY

\footnotetext{
${ }^{*}$ Speaker.
} 


\section{Introduction}

Quark chirality imbalance and a strong magnetic field can both be present in heavy-ion collisions, and their coupling can result in the chiral magnetic effect (CME) [1]. Searches for chirality effects using event-by-event analyses have been investigated theoretically and experimentally [2]. For Au+Au collisions at STAR, we use a three-point correlator $\gamma$ (see Section 2.1) to measure the fluctuations in the charge separation magnitudes, and use difference between the opposite-sign (OS) and the same-sign (SS) charged-particle pairs to suppress common backgrounds. Recent studies have indicated that a considerable amount of flow-related background remains in the $\gamma$ correlator [3, 4]. To quantify the range of the residual background, we use the $\kappa_{112}$ parameter (see Section 2.1) to estimate the expected range of known background levels from AMPT simulations (v2.21, string melting, hadronic scattering turned on, charge not strictly conserved [5]).

The isobaric collisions, such as ${ }_{44}^{96} \mathrm{Ru}+{ }_{44}^{96} \mathrm{Ru}$ and ${ }_{40}^{96} \mathrm{Zr}+{ }_{40}^{96} \mathrm{Zr}$, are proposed to disentangle the flow-related background and the real CME signals [6]. In these two collision systems, the background (elliptic flow) will be similar, while the "driving-force" of the CME, the initial magnetic field, will differ significantly.

In Section 2.1 we describe the observables and analysis details. In Section 2.2, we present correlation measurements for charged hadrons and identified particles $(\pi, K, p)$ from $\mathrm{Au}+\mathrm{Au}$ collisions. For the upcoming isobaric collision program at STAR, we make a projection of the CME signal (see Section 3) based on the theoretical calculation of the initial magnetic field and eccentricity in $\mathrm{Ru}+\mathrm{Ru}$ and $\mathrm{Zr}+\mathrm{Zr}$ collisions.

\section{Particle correlation in Au+Au collisions}

\subsection{Observables and data}

The three-point $\gamma$ correlator [6] is defined as follows,

$$
\begin{aligned}
\gamma & \left.\equiv \cos \left(\phi_{a}+\phi_{b}-2 \Psi_{\mathrm{RP}}\right)\right\rangle \\
& =\left\langle\cos \left(\phi_{a}+\phi_{b}-2 \Psi_{2}\right)\right\rangle /\left\langle\cos \left(2 \Psi_{2}-2 \Psi_{\mathrm{RP}}\right)\right\rangle \\
& =\left\langle\cos \left(\phi_{a}+\phi_{b}-2 \phi_{c}\right)\right\rangle / \nu_{2, c},
\end{aligned}
$$

which is sensitive to charge separation across the reaction plane in heavy-ion collisions. The $\phi_{a}$ and $\phi_{b}$ are the azimuthal angles of a correlated particle pair, and $\Psi_{2}$ and $\phi_{c}$ are used to approximate the reaction plane, $\Psi_{\mathrm{RP}}$. The $v_{2, c}$ is the elliptic flow, $v_{2}$, of the third particle $c$. A finite $\gamma_{\mathrm{OS}}-\gamma_{\mathrm{SS}}$, which quantifies the event-by-event fluctuation of charge distribution asymmetry, will indicate a possible charge separation across the reaction plane. Here "OS" denotes the particle pair with opposite charge sign and "SS" the same charge sign. For convenience, the notation $\Delta \gamma$ and $\gamma_{\mathrm{OS}}-\gamma_{\mathrm{SS}}$ will be used interchangeably in the rest of the proceedings.

The flow-related background is known to contribute to the $\gamma$ correlation. To quantitatively estimate the flow background, a background model for $\gamma$ and the two-particle correlator $\delta \equiv$ $\left\langle\cos \left(\phi_{a}-\phi_{b}\right)\right\rangle$ has been proposed and a $H$ correlator has been introduced [28]:

$$
\begin{aligned}
\Delta \delta & \equiv\left\langle\cos \left(\phi_{a}-\phi_{b}\right)\right\rangle_{\mathrm{OS}-\mathrm{SS}}=\Delta F+\Delta H \\
\Delta \gamma & \equiv\left\langle\cos \left(\phi_{a}+\phi_{b}-2 \Psi_{\mathrm{RP}}\right)\right\rangle_{\mathrm{OS}-\mathrm{SS}}=\kappa v_{2} \Delta F-\Delta H,
\end{aligned}
$$


where $\Delta F$ and $\Delta H$ are the amounts of charge separation resulting from the flow-related background and magnetic field, respectively. " $\Delta$ " refers to difference between "OS" and "SS" correlations. $\kappa$ quantifies the coupling between $v_{2}$ and $\delta$, which contaminates the $\gamma$ measurements [7]:

$$
\kappa=\frac{\Delta \gamma+\Delta H}{v_{2}(\Delta \delta-\Delta H)} .
$$

We introduce the $\kappa_{112}$ parameter (this is the $\kappa_{K}$ parameter in the talk slides) by setting $\Delta H$ to be zero for a maximum background scenario $\left.\kappa_{112} \equiv \kappa\right|_{\Delta H=0}=\frac{\Delta \gamma}{v_{2} \Delta \delta}$. The $\kappa_{112}$ parameter can be compared with $\kappa$ from background models such as AMPT simulations. Any excess of measured $\kappa_{112}$ for particle pairs of interest over the expected range from background models could indicate the presence of physics beyond known background, e.g., the CME.

For identified particle correlation measurements, $\sim 200$ million minimum-bias (MB) events of $\mathrm{Au}+\mathrm{Au}$ collisions at $\sqrt{s_{N N}}=200 \mathrm{GeV}$ from RHIC Run11 were used. Tracks reconstructed in the Time-Projection Chamber (TPC) [29, 30] with transverse momentum $p_{T}$ from 0.15 to $2.0 \mathrm{GeV} / \mathrm{c}$ were used for the estimation of the second-order event plane. Particle species $(\pi, K, p)$ were identified by applying cuts on the energy loss $(d E / d x)$ in the TPC. Moreover, the particle velocity $\beta$ measured via the Time-Of-Flight (TOF) [31] detector helps further select the desired particles.

\subsection{Identified particle correlation in Au+Au collisions}

Figure 1a shows the $\kappa_{112}$ measurements for charged hadrons in 7.7, 19.6, 39, and $200 \mathrm{GeV}$ $\mathrm{Au}+\mathrm{Au}$ collisions [3]. We are using the AMPT $(|\eta|<1.5) h^{ \pm} h^{ \pm}$correlation result for $\mathrm{Au}+\mathrm{Au}$ collisions at $200 \mathrm{GeV}$ as a background reference without the CME and it shows a weak centrality dependence between values of 1 and 2. STAR data for energies from 19.6 to $200 \mathrm{GeV}$ show very similar magnitudes of $\kappa_{112}$, well above the AMPT estimation of background levels for $200 \mathrm{GeV}$ in mid-peripheral and mid-central collisions. Results at $7.7 \mathrm{GeV}$ fall into the estimated background range. STAR data [8] showed that in peripheral collisions an additional short or intermediaterange background correlation also contributes to the $\gamma$, which may finally lead to the rise in $\kappa_{112}$ in peripheral collisions. With additional data from STAR BES II (Beam Energy Scan II) program, the errors will be reduced as estimated in Figure $1 \mathrm{~b}$.

For correlations involving identified particles, Fig. 2 shows $\Delta \gamma$ measurements of particle pairs $(\pi \pi, p K, \pi K, p p, p \pi)$ as functions of centrality. The $p p$ correlation magnitude is significantly larger than the other pairs. However, $\kappa_{112}$ (see Fig. $2 b$ ) for these particle pairs indicates a very different behavior. Only $\pi \pi$ correlation has $\kappa_{112}$ values larger than the AMPT background level $(\sim 1-2$, estimated from charged hadrons) in mid-peripheral and mid-central collisions, while the other pairs are falling within the range of 1 to 2 except the $p p$ pairs. $\kappa_{112}$ for $p p$ is found to be lower than 1 in those centrality bins. These results suggest that $\pi \pi$ correlation may dominate the $h^{ \pm} h^{ \pm}$charge separation signal. The other particle pairs will require more simulation events (the current statistics are limited by speed of AMPT simulations) to determine the background. The charge separation and background level for $p p$ pairs will require further investigation.

\section{Isobaric collision projection}

In spite of the observed sizable difference between same-sign and opposite-sign $\gamma$ correlations in $\mathrm{Au}+\mathrm{Au} 200 \mathrm{GeV}$ collisions, the interpretation of the results still remain controversial. Studies [9, 


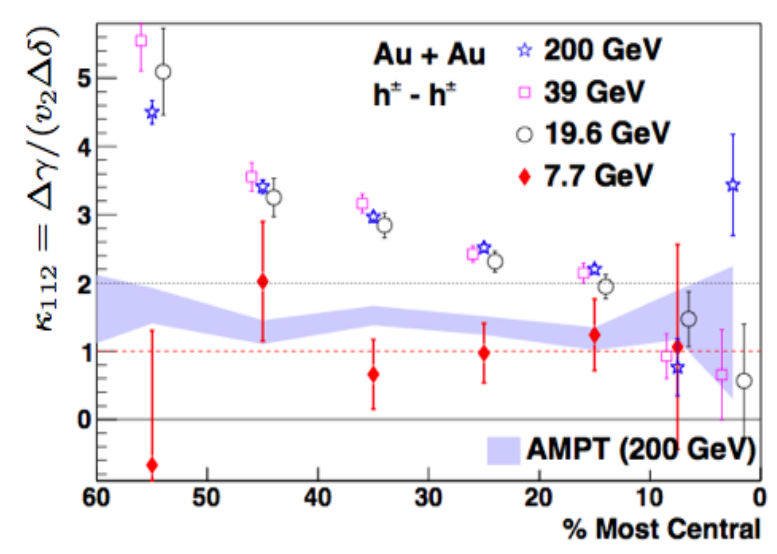

(a)

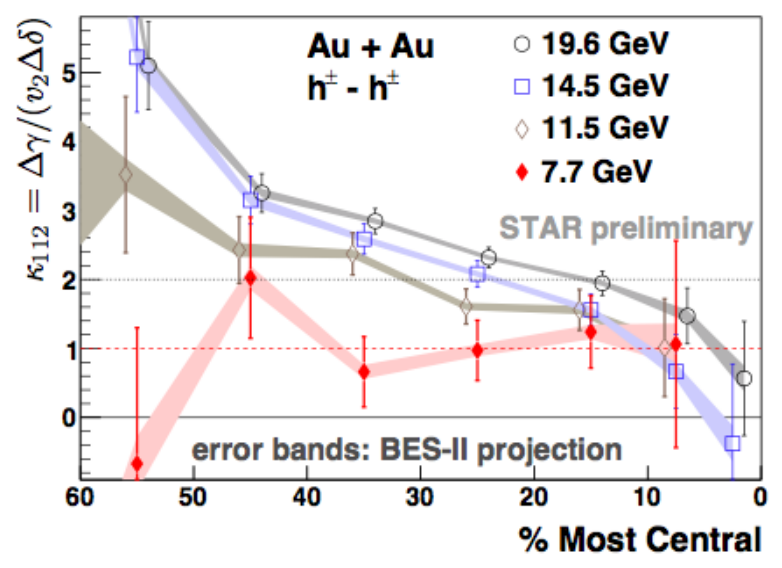

(b)

Figure 1: (a) $\kappa_{112}$ for $h^{ \pm} h^{ \pm}$in Au+Au collisions at 7.7, 19.6, 39, $200 \mathrm{GeV}$, and AMPT Au+Au $200 \mathrm{GeV}$ simulations. (b) $\kappa_{112}$ error projection (error bands) for the Beam Energy Scan phase II. The data points and error bars are from Beam Energy Scan Phase I.

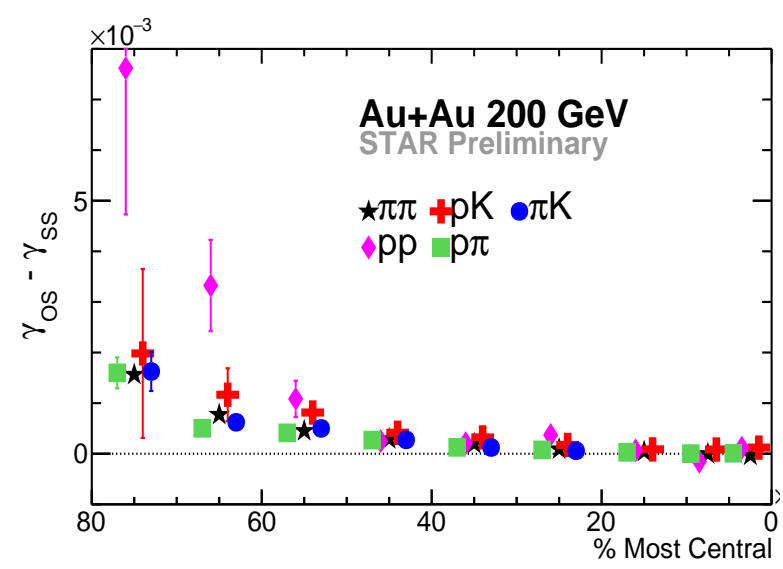

(a)

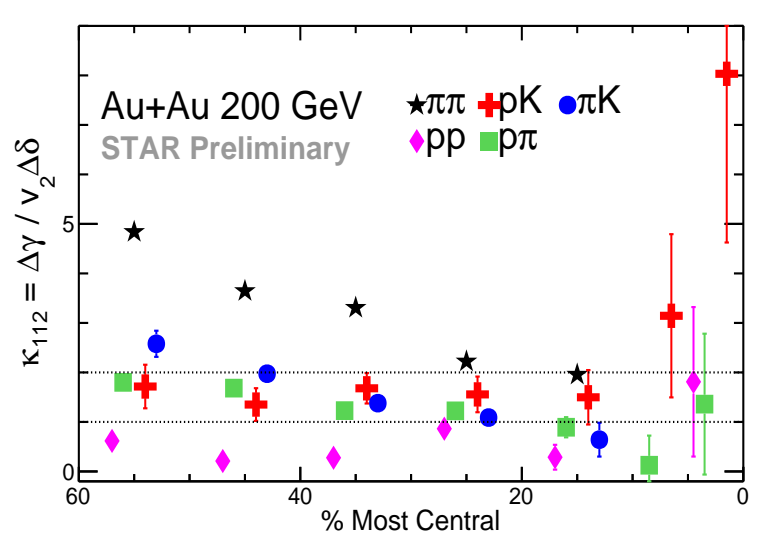

(b)

Figure 2: (a) $\gamma_{\mathrm{OS}}-\gamma_{\mathrm{SS}}$ and (b) $\kappa_{112}$ for $\pi \pi, p K, \pi K, p p$, and $p \pi$ as functions of centrality in $\mathrm{Au}+\mathrm{Au}$ collisions at $200 \mathrm{GeV}$.

$10,11,12,13]$ show that some conventional physics mechanisms such as local charge conservation and transverse momentum conservation could couple with the elliptic flow to contribute to $\Delta \gamma$ signals.

One possible strategy to disentangle flow-related backgrounds and the real CME signal is to see how the signal varies in two collision systems where the background (elliptic flow) is similar, but the signal "driving forces" (initial magnetic fields) are significantly different. Isobaric collisions, for example, ${ }_{44}^{96} \mathrm{Ru}+{ }_{44}^{96} \mathrm{Ru}$ and ${ }_{40}^{96} \mathrm{Zr}+{ }_{40}^{96} \mathrm{Zr}$, have been proposed [14] to potentially achieve this goal. The Glauber model simulation demonstrates the multiplicity distributions in these two systems are almost identical [18] except for 5-10\% central collisions, so the background contribution due to the multiplicity can be ignored for the CME analysis, in which only the 20-60\% central 
collisions will be our focus.

In the theoretical calculation [18], the spatial distribution of nucleons in either ${ }_{44}^{96} \mathrm{Ru}$ or ${ }_{40}^{96} \mathrm{Zr}$ in the rest frame can be written in the Woods-Saxon form:

$$
\rho(r, \theta) \propto \frac{1}{1+\exp \left[\left(r-R_{0}-\beta_{2} R_{0} Y_{2}^{0}(\theta)\right) / a\right]},
$$

where $R_{0}$ and $a$ represent the "radius" of the nucleus and the surface diffuseness parameter, respectively. The $\beta_{2}$ is the deformity of the nucleus. The e-A experiments $[19,20]$ and comprehensive model deduction [21] present two different sets of nucleus structure parameters for $\mathrm{Ru}$ and $\mathrm{Zr}$ as shown in Table. 1. In the rest of these proceedings, the set of parameters from e-A experiment will be referred to as case 1 , and the one from the comprehensive model deduction will be referred to as case 2 . The $\beta_{2}$ of $\mathrm{Ru}$ is larger than $\mathrm{Zr}$ ( $\mathrm{Ru}$ is more deformed) in case 1 , whereas it is the opposite in case 2 . This systematic uncertainty impacts the multiplicity distribution negligibly, but may exert noticeable influence on the CME signal (via the magnetic field) and the $v_{2}$ driven backgrounds (via $\varepsilon_{2}$, the initial spatial eccentricity of the participant zone).

\begin{tabular}{ccccc}
\hline \hline Sources & Nucleus & $R_{0}$ & $\mathrm{a}$ & $\beta_{2}$ \\
\hline Case 1 & ${ }_{44}^{96} R u$ & 5.085 & 0.46 & 0.158 \\
(e-A Experiment) & ${ }_{40}^{96} \mathrm{Zr}$ & 5.02 & 0.46 & 0.08 \\
\hline Case 2 & ${ }_{44}^{96} R u$ & 5.085 & 0.46 & 0.053 \\
(Comprehensive Model Deduction) & ${ }_{40}^{96} \mathrm{Zr}$ & 5.02 & 0.46 & 0.217 \\
\hline
\end{tabular}

Table 1: Woods-Saxon Parameters.

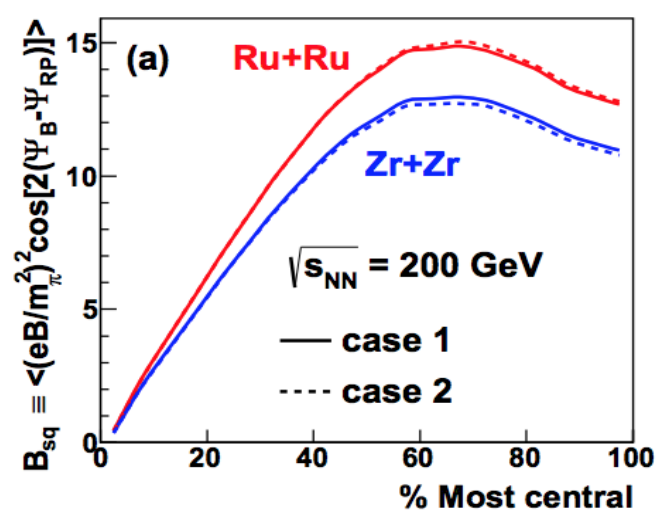

(a)

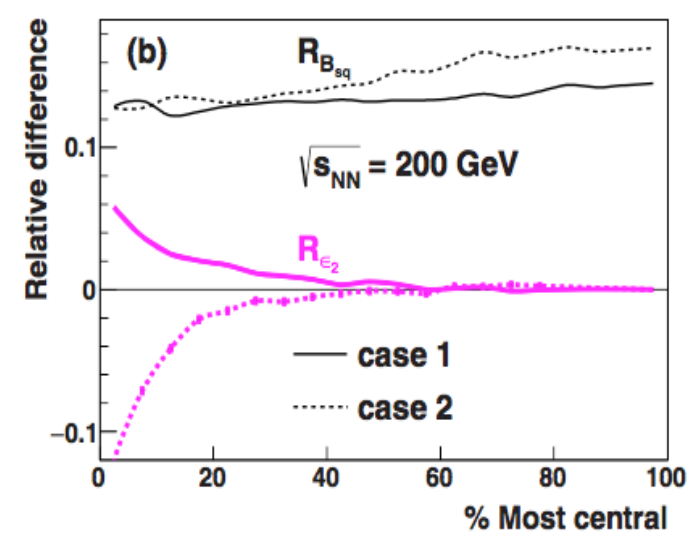

(b)

Figure 3: (a) Initial magnetic field squared with correction from azimuthal fluctuation $\left(B_{s q}\right)$ in $\mathrm{Ru}+\mathrm{Ru}$ and $\mathrm{Zr}+\mathrm{Zr}$ collisions at $200 \mathrm{GeV}$. (b) The relative difference of $B_{s q}$ and $\varepsilon_{2}$ (initial eccentricity). Solid and dotted lines represent case 1 and case 2 parameter sets. 


\subsection{Initial magnetic field and eccentricity}

The different charges carried by these two kinds of nuclei result in different initial magnetic fields (mostly generated by spectator protons) [22, 23], which finally produce distinctive magneticfield driven charge separation signals in these two collision systems. Figure 3 shows the theoretical calculation of the initial magnetic field squared with correction from azimuthal fluctuation of the magnetic field orientation $\left(B_{s q}=\left\langle\left(e B / 2 m_{\pi}^{2}\right)^{2} \cos \left[2\left(\psi_{\mathrm{B}}-\psi_{\mathrm{RP}}\right)\right]\right\rangle\right)$ as a function of centrality in $\mathrm{Ru}+\mathrm{Ru}$ and $\mathrm{Zr}+\mathrm{Zr}$ collisions at $\sqrt{s_{N N}}=200 \mathrm{GeV}$, as well as their relative difference. The $B_{s q}$ quantifies the capability of the magnetic field to drive charge separation signal in the correlator $\gamma$ $[26,27]$. Most of the systematic uncertainty can be canceled by their relative difference $\left(R_{B_{s q}}\right.$, in the following text, we use $R_{F} \equiv 2\left(F^{\mathrm{Ru}+\mathrm{Ru}}-F^{\mathrm{Zr}+\mathrm{Zr}}\right) /\left(F^{\mathrm{Ru}+\mathrm{Ru}}+F^{\mathrm{Zr}+\mathrm{Zr}}\right)$, where $\mathrm{F}$ can be $B_{s q}, \varepsilon_{2}$ or $S$ to denote "relative difference" of this quantity between $\mathrm{Ru}+\mathrm{Ru}$ and $\mathrm{Zr}+\mathrm{Zr}$ collision systems). We can observe $R_{B_{s q}}$ increases slowly from $13 \%$ to $15 \%$ (18\%) as the collision becomes more peripheral. The nucleus deformity uncertainty has more influence in peripheral collisions. In addition, the relative difference of the initial eccentricity $\varepsilon_{2}$ is also plotted on Fig. 3b, which is consistent with zero in peripheral collisions. In central collisions, however, these parameter sets for the two cases deviate significantly. Since the relative difference in $v_{2}$ should be very similar to $R_{\varepsilon_{2}}$, we can deduce that in these two collision systems, their $v_{2}$ values will stay almost the same for $20 \%-60 \%$ events. Also, the measurement of the relative difference of elliptic flow in central isobaric collisions may provide a definite answer to the question: which nucleus is more deformed?

\subsection{Charge separation signal projection}

Given the initial magnetic field and eccentricities, the magnetic field driven charge separation contribution to the charge separation $\left(\mathrm{S}=\Delta \gamma \times N_{\text {part }}\right)$ can be estimated. The signal in these two collision systems can be written [18]:

$$
\begin{aligned}
S^{\mathrm{Ru}+\mathrm{Ru}} & =\bar{S}\left[(1-b g)\left(1+\frac{R_{B_{s q}}}{2}\right)+b g\left(1+\frac{R_{\varepsilon_{2}}}{2}\right)\right] \\
S^{\mathrm{Zr}+\mathrm{Zr}} & =\bar{S}\left[(1-b g)\left(1-\frac{R_{B_{s q}}}{2}\right)+b g\left(1-\frac{R_{\varepsilon_{2}}}{2}\right)\right]
\end{aligned}
$$

where $b g \in[0,1]$ denotes the background contribution from elliptic flow and $\bar{S}=\left(S^{\mathrm{Ru}+\mathrm{Ru}}+S^{\mathrm{Zr}+\mathrm{Zr}}\right) / 2$. Then the relative difference of $\mathrm{S}$ can be expressed by:

$$
R_{S}=(1-b g) R_{B_{s q}}+b g R_{\varepsilon_{2}}
$$

Figure 4a (Figure 4b) shows the projected signal S, with 1.2 billion minimum-bias events and $67 \%$ (80\%) background level (percentage of $\Delta \gamma$ caused by the $v_{2}$ ) as a function of centrality in $\mathrm{Ru}+\mathrm{Ru}$ and $\mathrm{Zr}+\mathrm{Zr}$ collisions at $200 \mathrm{GeV}$. The relative difference is shown in Fig. 4c (Fig. 4d), where $R_{S}$ and $R_{\varepsilon_{2}}$ display different trends. This feature promises a chance to quantify the CME signals even with a high level of flow-related background. As a function of background level [18], the CME signal and its significance are plotted in Fig. 5. Given 1.2 billion minimum-bias events and $80 \%$ background, the significance of the CME signal reaches $5 \sigma$. With the same background level, but improved event plane resolution from a new STAR detector, the Event Plane Detector(EPD), the significance of the CME signal can be further raised to $7.8 \sigma$. 


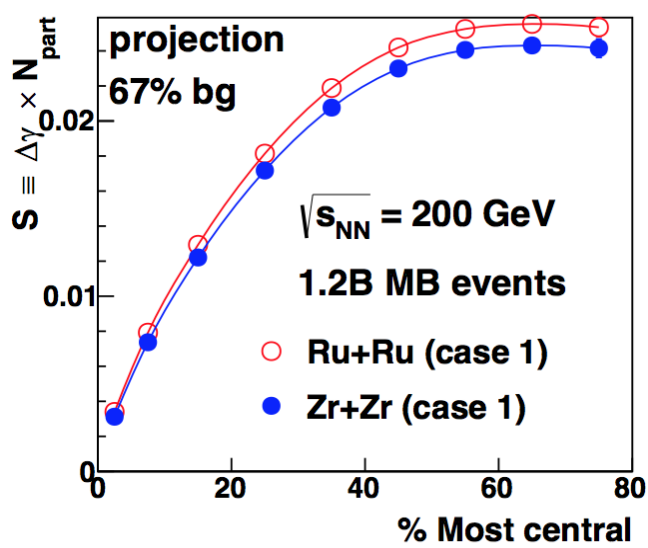

(a)

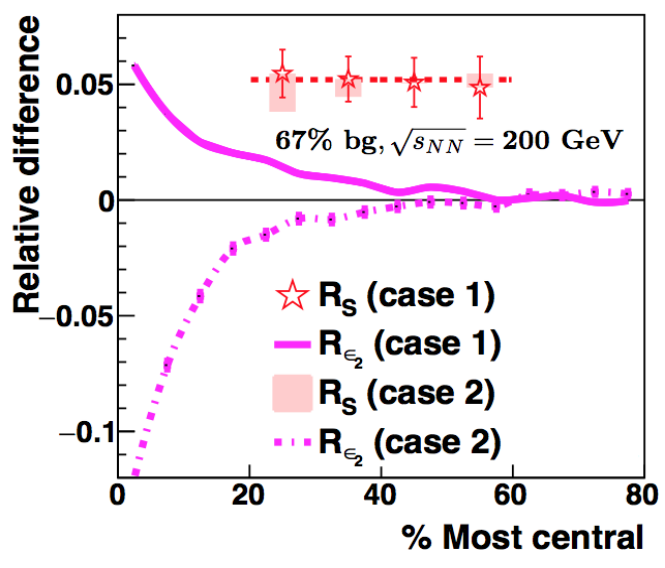

(c)

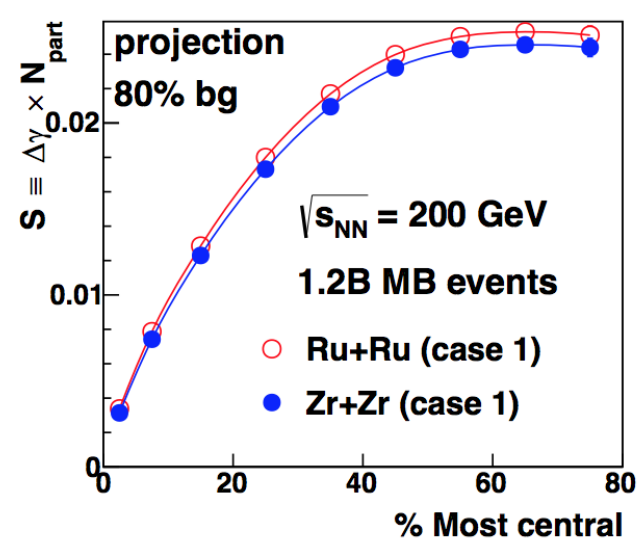

(b)

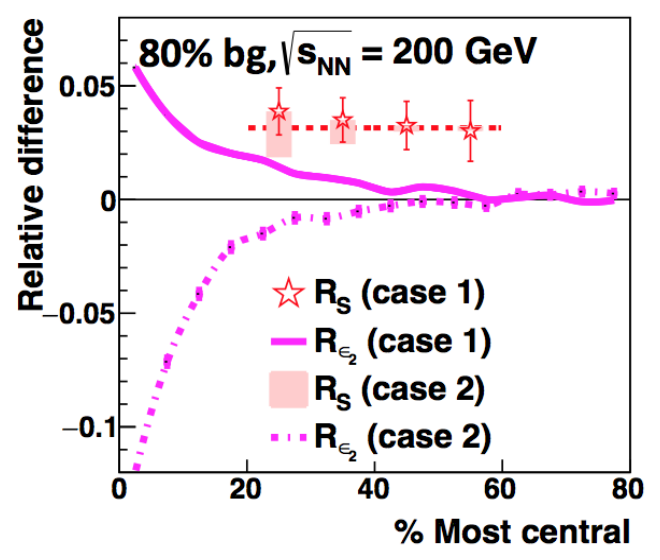

(d)

Figure 4: (a-b) The CME signal projection with 67\%(a) / 80\%(b) background level and 1.2 billion events in $\mathrm{Ru}+\mathrm{Ru}$ and $\mathrm{Zr}+\mathrm{Zr}$ collisions at $200 \mathrm{GeV}$. The nucleus structure parameters are from case1. (c-d) The Relative differences of the predicted signal and eccentricity between $\mathrm{Ru}+\mathrm{Ru}$ and $\mathrm{Zr}+\mathrm{Zr} 200 \mathrm{GeV}$ collisions in both cases, with 67\%(c) and 80\%(d) background level.

\section{Summary and outlook}

We have presented STAR measurements of $\gamma$ correlations and $\kappa_{112}$ parameters for identified particle pairs $(\pi \pi, p K, \pi K, p p, p \pi)$ in Au+Au collisions at $\sqrt{s_{N N}}=200 \mathrm{GeV}$. The $\kappa_{112}$ values for particle pairs $p K, \pi K, p \pi$ are between 1 and 2 . The $\kappa_{112}$ parameter for $\pi \pi$ correlations has a similar magnitude to that for charged hadrons in $\mathrm{Au}+\mathrm{Au}$ collisions at $200 \mathrm{GeV}$, which is larger than the background estimated from AMPT. The $p p$ correlations yield a $\kappa_{112}$ smaller than 1 .

Theoretical calculation of the initial magnetic field and eccentricity of $\mathrm{Ru}+\mathrm{Ru}$ and $\mathrm{Zr}+\mathrm{Zr}$ is reported. The results show that even with background level as high as $80 \%$, it is still possible to obtain a $5 \sigma$ significance of the magnetic field induced charge separation signal from 1.2 billion minimum-bias events at STAR. With improved event plane resolution of the EPD, the statistical error may be further reduced. Meanwhile, isobaric collision program can also discern which of the two nuclei $(\mathrm{Ru}$ and $\mathrm{Zr}$ ) is more deformed. 


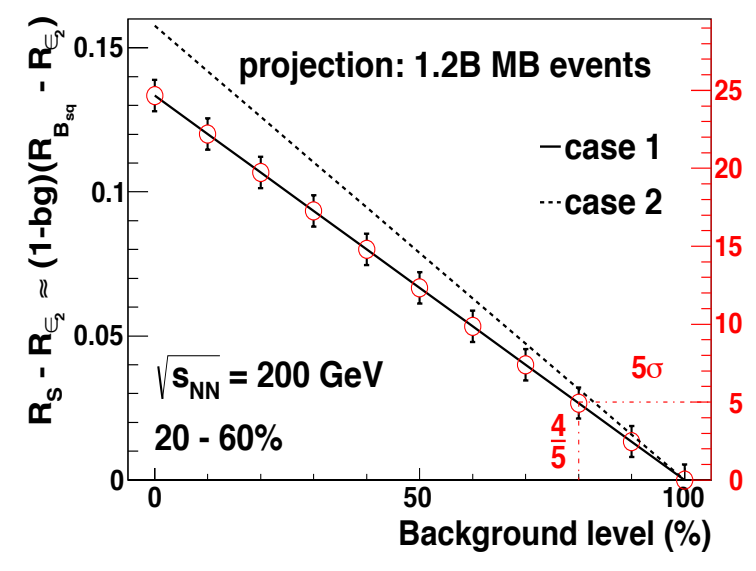

(a)

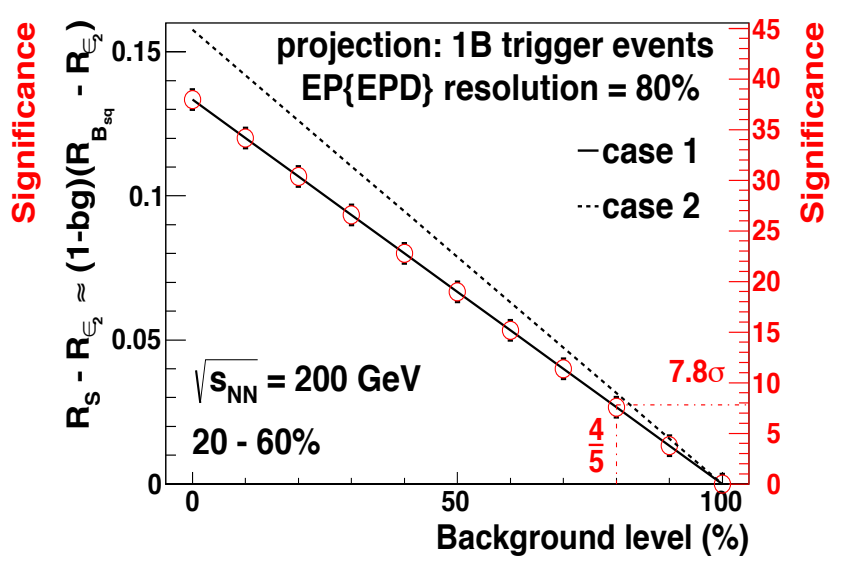

(b)

Figure 5: Projected signal magnitude and significance without (a) and with (b) EPD (Event-Plane Detector) as a function of background level.

\section{References}

[1] D. E. Kharzeev, L. D. McLerran, Warringa, H. J., Nucl. Phys. A 803, 227-253 (2008)

[2] D. E. Kharzeev, J. Liao, S. A. Voloshin, G. Wang, Prog. Part. Nucl. Phys. 88, 1-28 (2016).

[3] L. Adamczyk, et al., Phys. Rev. Lett. 113, 052302 (2014).

[4] V. Khachatryan, et al.Phys. Rev. Lett. 118, 122301 (2007).

[5] Z.-W. Lin, C. M. Ko, B.-A. Li, B. Zhang, S. Pal, Phys. Rev. C 72064901 (2005).

[6] S. A. Voloshin, Phys. Rev. C 70, 057901 (2004).

[7] L. Wen, Nuclear Physics A 967, 756-759 (2017).

[8] P. Tribedy, [for the STAR Collaboration]. arXiv:1704.03845 [nucl-ex]

[9] S. Pratt, arXiv:1002.1758 [nucl-th]

[10] S. Schlichting and S. Pratt, arXiv:1005.5341 [nucl-th]

[11] S. Schlichting and S. Pratt, Phys. Rev. C 83, 014913 (2011).

[12] S. Pratt, S. Schlichting and S. Gavin, Phys. Rev. C 84, 024909 (2011).

[13] A. Bzdak, V. Koch and J. Liao, Phys. Rev. C 83, 014905 (2011).

[14] S. A. Voloshin, Phys. Rev. Lett. 105, 172301 (2010).

[15] K. Adcox et al. [PHENIX Collaboration], Phys. Rev. Lett. 86, 3500 (2001).

[16] I. G. Bearden et al. [BRAHMS Collaboration], Phys. Lett. B 523, 227 (2001).

[17] B. B. Back et al. [PHOBOS Collaboration], Phys. Rev. C 65, 061901 (2002).

[18] W. -T. Deng, X. -G. Huang, G.-L. Ma and G. Wang, Phys. Rev. C 94, 041901 (2016).

[19] S. Raman, C. W. G. Nestor, Jr and P. Tikkanen, Atom. Data Nucl. Data Tabl. 78, 1 (2001). 
[20] B. Pritychenko, M. Birch, B. Singh and M. Horoi, Atom. Data Nucl. Data Tabl. 107, 1 (2016).

[21] P. Moller, J. R. Nix, W. D. Myers and W. J. Swiatecki, Atom. Data Nucl. Data Tabl. 59, 185 (1995).

[22] V. Skokov, A. Y. Illarionov and V. Toneev, Int. J. Mod. Phys. A 24, 5925 (2009).

[23] W. T. Deng and X. G. Huang, Phys. Rev. C 85, 044907 (2012).

[24] W. T. Deng and X. G. Huang, Phys. Rev. C 85, 044907 (2012).

[25] W. T. Deng and X. G. Huang, Phys. Lett. B 742, 296 (2015).

[26] J. Bloczynski, X. G. Huang, X. Zhang and J. Liao, Phys. Lett. B 718, 1529 (2013).

[27] J. Bloczynski, X. G. Huang, X. Zhang and J. Liao, Nucl. Phys. A 939, 85 (2015).

[28] A. Bzdak, V. Koch, J. Liao, Lecture Notes in Physics 871, 529 (2013).

[29] H. Wieman, et al., IEEE Trans. Nucl. Sci. NS-44, 671 (1997).

[30] M. Anderson, et al., The STAR Time Projection Chamber, Nucl. Instr. and Meth. A 499, 655 (2003).

[31] W. J. Llope et al., Nucl. Instrum. Methods Phys. Res., Sect. A, 522, 252âĂŞ273 (2004).

[32] C. Whitten Jr., AIP Conference Proceedings 980390 